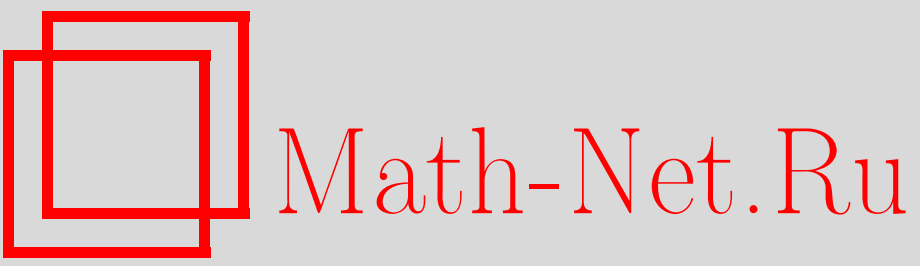

Е. И. Бунина, Элементарная эквивалентность унитарных линейных групп над кольцами и телами, УМН, 1998, том 53, выпуск 2, 137-138

DOI: https://doi.org/10.4213/rm32

Использование Общероссийского математического портала Math-Net.Ru подразумевает, что вы прочитали и согласны с пользовательским соглашением

http://www . mathnet.ru/rus/agreement

Параметры загрузки:

IP : 3.89 .185 .249

26 апреля 2023 г., 08:01:14 


\title{
ЭЛЕМЕНТАРНАЯ ЭКВИВАЛЕНТНОСТЬ УНИТАРНЫХ ЛИНЕЙНЫХ ГРУПП НАД КОЛЬЦАМИ И ТЕЛАМИ
}

\author{
Е. И. БунинА
}

Введение. В [5] А. И. Малшцев доказал следующую теорему: две линейные группы $G L_{m}(K)$ и $G L_{n}(L)$, где $K$ и $L-$ поля и $m, n \geqslant 3$, элементарно эквивалентны тогда и толшко тогда, когда $m=n$ и поля $K$ и $L$ элементарно эквивалентны. Прогресс в теории моделей позволил К. И. Бейдару и А. В. Михалеву предложить в [3] общий способ решения проблем такого типа. Они доказали подобные теоремы для групп над первичными ассоциативными кольцами, содержащими 1 и $1 / 2$, и над телами.

В [6] была доказана теорема, подобная теореме Мальцева, но для унитарных линейных групп над полями с инволюцией. Доказательство было построено "элементарньми" методами, аналогично доказательству Мальева. В данной работе получены теоремы для унитарных групп над ассоциативными кольцами с инволюцией, содержащими 1 и $1 / 2$, а также над телами. Они доказаны с помощью теорем [3] К. И. Бейдара и А. В. Михалева, а также И. З. Голубчика и А. В. Михалева [1].

\section{1. Формулировки основных теорем.}

ОПРЕДЕЛЕНИЕ 1 . Если $K$ - кольцо с инволюцией $j$, то будем обозначать через $\tau$ инволюцию кольца матриц над $K\left(M_{2 n}(K)\right)$ вида $\tau: A \mapsto Q_{2 n} \circ\left(A^{j}\right)^{T} \circ Q_{2 n}^{-1}$, где $Q_{2 n}$ - форма максимального ранга, приведенная к каноническому виду.

Теорема 1. Если $K_{1}$ и $K_{2}$ - ассоциативные (коммутативные) кольца, содержащие $1 / 2$ и 1/3, $j_{1}$ и $j_{2}$ - инволючии в кольцах $K_{1}$ и $K_{2}$ соответственно, $n, m>2$ $(n, m>1), Q_{2 n}$ и $Q_{2 m}$ - формы максимального ранга, то унитарные линейнье группьи $U_{2 n}\left(K_{1}, j_{1}, Q_{2 n}\right)$ и $U_{2 m}\left(K_{2}, j_{2}, Q_{2 m}\right)$ әлементарно әквивалентны тогда и только тогда, когда $m=n$ и $M_{2 n}\left(K_{1}\right)$ и $M_{2 m}\left(K_{2}\right)$ әлементарно әквивалентны как кольца $c$ инволючиями $\tau_{1}$ и $\tau_{2}$ соответственно.

Теорема 2. Если тела (поля) $F_{1}$ и $F_{2}$ имеют характеристику, больиую $2, j_{1}$ и $j_{2}$ - инволюиии в телах $F_{1}$ и $F_{2}$ соответственно, $n, m>2(n, m>1), Q_{2 n}$ и $Q_{2 m}$ - формы максимального ранга, то унитарные линейные группы $U_{2 n}\left(F_{1}, j_{1}, Q_{2 n}\right)$ u $U_{2 m}\left(F_{2}, j_{2}, Q_{2 m}\right)$ әлементарно әквивалентны тогда и только тогда, когда тела (поля) $F_{1}$ и $F_{2}$ әлементарно әквивалентны как тела (поля) с инволючиями $j_{1}$ и $j_{2}$ соответственно.

\section{2. Основные леммы.}

Лемма 1. Если кольца $K$ и $S$ ассочиативны, содержат $1 / 2$ и центры колец содержат әлементы $\alpha$ и $\beta$ соответственно, такие, что $\alpha \cdot \alpha^{j}-1 u \beta \cdot \beta^{j^{\prime}}-1$ обратимьь, $n \geqslant 3$, и $U_{2 n}\left(K, j, Q_{2 n}\right)$ изоморфна $U_{2 m}\left(S, j^{\prime}, Q_{2 m}\right)$, то кольцо $M_{2 n}(K)$ изоморфно кольиу $M_{2 m}(S)$ и существует такой изоморфизм $A$ колеи, $M_{2 n}(K)$ и $M_{2 m}(S)$, что на $E_{f}(R, \tau)$ он совпадает с изоморфизмом групп $U_{2 n}\left(K, j, Q_{2 n}\right)$ и $U_{2 m}\left(S, j^{\prime}, Q_{2 m}\right)$.

Аналогичную лемму можно сформулировать, если кольца $K$ и $S$ коммутативны и $n \geqslant 2$ :

ЛЕмма 2. Если кольца $K$ и $S$ ассоциативны и коммутативнь, содержат $1 / 2$ и әлементы $\alpha$ и $\beta$ соответственно такие, что $\alpha \cdot \alpha^{j}-1 u \beta \cdot \beta^{j}-1$ обратимь, $n \geqslant 2$, и $U_{2 n}\left(K, j, Q_{2 n}\right)$ изоморфна $U_{2 m}\left(S, j^{\prime}, Q_{2 m}\right)$, то кольцо $M_{2 n}(K)$ изоморфно кольцу $M_{2 m}(S)$ и существует такой изоморфизм $A$ колец $M_{2 n}(K)$ и $M_{2 m}(S)$, что на $E_{f}(R, \tau)$ он совпадает с изоморфизмом групп $U_{2 n}\left(K, j, Q_{2 n}\right)$ u $U_{2 m}\left(S, j^{\prime}, Q_{2 m}\right)$.

Лемма 3. Изоморфизм колеи, $M_{2 n}(K)$ и $M_{2 m}(S)$ из лемм 1 и 2 сохраняет инволюиию $\tau$, т.е. диаграмма

коммутативна.

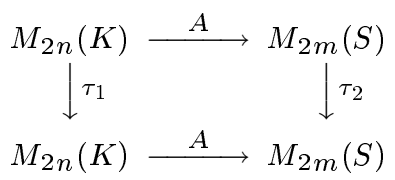


ЛЕмма 4. Пусть $F_{1}$ и $F_{2}$ - тела характеристики, больией 2 , с инволюииями $j_{1}$ и $j_{2}$ соответственно; $M_{2 n}\left(F_{1}, \tau_{1}\right)$ и $M_{2 n}\left(F_{2}, \tau_{2}\right)$ - кольца матрич, размера $n \times n$ над $F_{1}$ и $F_{2}$ с инволюциями $\tau_{1}$ и $\tau_{2}$ соответственно, получающимися из инволючий $j_{1}$ и $j_{2}$ следующим образом:

$$
\left(a_{i j}\right)^{\tau_{1}}=Q_{2 n}\left(a_{j i}^{j_{1}}\right) Q_{2 n}^{-1} ; \quad\left(a_{i j}\right)^{\tau_{2}}=Q_{2 n}\left(a_{j i}^{j_{2}}\right) Q_{2 n}^{-1} .
$$

Пусть, кроме того, существует изоморфизм $A$ межуду $M_{2 n}\left(F_{1}, \tau_{1}\right)$ и $M_{2 n}\left(F_{2}, \tau_{2}\right)$, коммутирующий с инволючией, т.е. такой, что $\forall f \in M_{2 n}\left(F_{1}, \tau_{1}\right) \quad A\left(f^{\tau_{1}}\right)=A(f)^{\tau_{2}}$.

Тогда существует изоморфизм $B$ тел $F_{1}$ и $F_{2}$ такой, что $\forall a \in F_{1} B\left(a^{j_{1}}\right)=B(a)^{j_{2}}$.

3. Доказательство основных теорем. В [3] была доказана следующая теорема. Пусть $U, V$ и $W$ - абстрактные классы алгебраических систем сигнатур $\Omega_{U}, \Omega_{V}$ и $\Omega_{W}$ соответственно, замкнутые относительно взятия ультрастепеней, $F=\left\{F_{i} \mid i=1,2, \ldots\right\}$ и $G=\left\{G_{i j} \mid\right.$ $i, j=1,2, \ldots\}$ - регулярные семейства отображений из $U$ в $V$ и в $W$ соответственно. Пусть существует натуральное $m$ такое, что для любых алгебраических систем $R, S \in U$ и любых целых $p, q>m$ соотношение $F_{p}(R) \cong F_{q}(S)$ эквивалентно существованию таких номеров $r, s \geqslant 1$, что $G_{r p}(R) \cong G_{s q}(S)$. Пусть теперь $R, S \in U$ и $p, q \geqslant m$. Тогда $F_{p}(R) \equiv F_{q}(S)$, если и только если существуют номера $r, s \geqslant 1$ такие, что $G_{r p}(R) \equiv G_{s q}(S)$. Теперь мы можем доказать теоремы, сформулированные в п. 1 .

ДОКАЗАТЕЛЬСТво ТЕОРЕМЫ 1 . Пусть $U$ - это класс ассоциативных колец, содержащих $1 / 2$ и $1 / 3$, с инволюциями, $V$ - класс групп, $W=U, F=\left\{U_{6}(\cdot), U_{8}(\cdot), \ldots\right\}, G=$ $\left\{\left(M_{6}(\cdot), \tau(\cdot)\right) ;\left(M_{8}(\cdot), \tau(\cdot)\right) ; \ldots\right\}$ - семейства отображений из $U$ в $V$ и $W$ соответственно. Очевидно, что оба семейства содержат регулярные отображения и классы $U, V, W$ замкнуты относительно ультрастепеней. По ранее доказанному $U_{2 n}\left(K_{1}, j_{1}\right) \cong U_{2 m}\left(K_{2}, j_{2}\right)(n, m>2)$ тогда и только тогда, когда $\left(M_{2 n}\left(K_{1}\right), \tau_{1}\right) \cong\left(M_{2 m}\left(K_{2}\right), \tau_{2}\right)$ как кольца с инволюциями. Отсюда и следует утверждение теоремы для некоммутативных колец.

Утверждение теоремы для коммутативных колец доказывается совершенно аналогично, только следует рассматривать класс коммутативных колец, содержащих $1 / 2$ и $1 / 3$, и $n, m>1$ вместо $n, m>2$.

ДОКАЗАТЕЛЬСТВО ТЕОРЕМЫ 2. Пусть $U$ - класс тел с инволюцией характеристики, болшей $2, V$-класс групп, $W=U, F=\left\{U_{6}\left(\cdot, \cdot, Q_{6}\right), U_{8}\left(\cdot, \cdot, Q_{8}\right), \ldots\right\}, G=\{G\}$ - тождественное отображение из $U$ в $W$. Очевидно, что семейства $F$ и $G$ содержат регулярные отображения из $U$ в $V$ и $W$ соответственно и классы $U, V$ и $W$ замкнуты относительно взятия ультрастепеней. Из ранее доказанного следует, что $U_{2 n}\left(F_{1}, j_{1}, Q_{2 n}\right) \cong U_{2 m}\left(F_{2}, j_{2}, Q_{2 m}\right)$ тогда и только тогда, когда $n=m$ и $\left(F_{1}, j_{1}\right) \cong\left(F_{2}, j_{2}\right)$, откуда очевидным образом следует утверждение теоремы для тел.

Утверждение теоремы для полей доказывается аналогично предыдущему, нужно лишь заменить $U$ на класс полей с инволюцией характеристики, большей 2 , и рассматривать $n, m>1$ вместо $n, m>2$.

\section{СПИСОК ЛИТЕРАТУРЫ}

[1] Голубчик И. З., Михалев А. В. // Зап. научн. семин. ЛОМИ. 1983. Т. 132. С. 97-109. [2] Бэр Р. Линейная алгебра и проективная геометрия. М.: Наука, 1995. [3] Beidar C. I., Michalev A. V. // Contemp. Math. 1992. V. 131. Р. 29-35. [4] Мальцев А. И. Алгебраические системы. М.: Наука, 1970. [5] Мальцев А. И. Об элементарных свойствах линейных групп // Проблемы математики и механики. Новосибирск, 1961. С. 110-132. [6] Бунина Е. И. Элементарная эквивалентность унитарных линейных групп над полями // Фундаментальная и прикладная математика, 1998 (в печати). 